Kyushu Sangyo University

\title{
AN EXTENSION OF JÓNSSON-TARSKI REPRESENTATION AND MODEL EXISTENCE IN PREDICATE NON-NORMAL MODAL LOGICS
}

\author{
YOSHIHITO TANAKA
}

\begin{abstract}
In this paper, we give an extension of the Jónsson-Tarski representation theorem for both normal and non-normal modal algebras so that it preserves countably many infinitary meets and joins. To extend the JónssonTarski representation to non-normal modal algebras we consider neighborhood frames instead of Kripke frames just as Došen's duality theorem for modal algebras, and to deal with infinite meets and joins, we make use of Q-filters instead of prime filters. Then, we show that every predicate modal logic, whether it is normal or non-normal, has a model defined on a neighborhood frame with constant domains, and give completeness theorem for some predicate modal logics. We also show the same results for infinitary modal logics.
\end{abstract}

\section{INTRODUCTION}

In this paper, we give an extension of the Jónsson-Tarski representation theorem for both normal and non-normal modal algebras so that it preserves countably many infinitary meets and joins. To extend the Jónsson-Tarski representation to nonnormal modal algebras we consider neighborhood frames instead of Kripke frames just as Došen's duality theorem for modal algebras [5], and to deal with infinite meets and joins, we make use of Q-filters instead of prime filters. Then, we show a model existence theorem for every predicate modal logic and completeness theorem for some predicate modal logics. We also show the same results for infinitary modal logics. Since we deal with non-normal modal algebras, we call a Boolean algebra with unary operator $\square$ by modal algebra, as does Došen in [5, and call a modal algebra which satisfies $\square 1=1$ and $\square(x \wedge y)=\square x \wedge \square y$ by normal modal algebra. We define modal logics and normal modal logics in the same manner. That is, the class of modal logics in this paper includes both normal and non-normal modal logics.

It is well known as the Jónsson-Tarski representation [6 that for any normal modal algebra $A$, there exists a monomorphism from $A$ to the dual algebra of the Kripke frame defined by the set of all prime filters of $A$ and the binary relation obtained from the modal operator of $A$. The Jónsson-Tarski representation is a strong tool in the researches of propositional normal modal logics [4, 3], but the naive application of these researches to predicate modal logics does not work, as the embedding does not preserve infinite meets and joins.

In the case of Boolean algebras, Rasiowa-Sikorski [9, 10 introduced the notion of Q-filters to strengthen the notion of prime filters and showed the Rasiowa-Sikorski lemma, which states that two distinct points in a Boolean algebra can be separated by a Q-filter. From the Rasiowa-Sikorski lemma, it follows that for any Boolean algebra $A$ and any countable subset $S$ of $\mathcal{P}(A)$, there exists an embedding $f$ from $A$ into the power set algebra of the set of all Q-filters of $A$, which satisfies that $f(\bigwedge X)=\bigwedge f[X]$ and $f(\bigvee X)=\bigvee f[X]$ for all $X \in S$. Applying this result to the Lindenbaum algebra, an algebraic proof of the completeness theorem for

Key words and phrases. Jónsson-Tarski representation, Neighborhood model, Modal logic, Predicate logic, Infinitary logic. 
the classical predicate logic is obtained [9, 10. In the case of Heyting algebras, Rauszer-Sabarski [1] showed that for any Heyting algebra $A$ and any countable subset $S \subseteq \mathcal{P}(A)$ which satisfies certain conditions, there exists an embedding $f$ from $A$ into the dual Heyting algebra of the intuitionistic Kripke frame defined on the set of all Q-filters of $A$, which preserves all infinite meets and joins in $S$. One of the conditions for the existence of the embedding is

$$
\bigwedge_{x \in X}(x \vee y)=\bigwedge X \vee y
$$

for any $X \in S$ and $y \in A$. In fact, Rauszer-Sabarski [12 proved that (1.1) is necessary for the Rasiowa-Sikorski lemma for Heyting algebras. When we interpret universal quantifiers in a formula as an infinite meets in Heyting algebras, (1.1) corresponds to the axiom

$$
\mathrm{CD}=\forall x(\phi \vee q) \supset(\forall x \phi) \vee q
$$

of constant domains. By using the Rasiowa-Sikorski lemma for Heyting algebras given in [12], Ono-Rauszer [8] showed that if CD is provable in a superintuitionistic $\operatorname{logic} \mathrm{L}$, there exists an intuitionistic Kripke model $\mathcal{M}$ with constant domains which satisfies that $\phi \in \mathrm{L}$ iff $\mathcal{M} \models \phi$ for any formula $\phi$.

Then, Tanaka-Ono 14 showed an extension of the Jónsson-Tarski representation for normal modal algebras which preserves countably many infinitary meets and joins: For any modal algebra $A$ and any countable subset $S \subseteq \mathcal{P}(A)$ which satisfies

$$
\square \text { ๑X }=\bigwedge_{x \in X} \square x
$$

for any $X \in S$ and some other conditions, there exists an embedding from $A$ to the dual algebra of the Kripke frame defined by the set of all Q-filters of $A$, which preserves all infinite meets and joins in $S$. The equation (1.2) corresponds to the Barcan formula

$$
\mathrm{BF}=\forall x \square \phi \supset \square \forall x \phi
$$

and its infinitary variant

$$
\mathrm{BF}_{\omega_{1}}=\bigwedge_{i \in \omega} \square p_{i} \supset \square \bigwedge_{i \in \omega} p_{i} .
$$

It is shown in 14 that for any universal propositional normal modal logic $\mathrm{L}$, the predicate modal logic defined by the least predicate extension of $L$ and BF is complete with respect to the class $C$ of Kripke frames with constant domains, where $C$ is the class of Kripke frames which validate L. It is also shown in 14 that the same result as predicate modal logics and BF holds for infinitary modal logics and $\mathrm{BF}_{\omega_{1}}$.

In this paper, we introduce a similar extension of the Jónsson-Tarski representation for both normal and non-normal modal algebras, without assuming (1.2). It is proved by Došen [5] that the category of modal algebras is dually equivalent to the category of neighborhood frames, and it is known that there exists a neighborhood frames which refutes (1.2) (see, [2, 7]), while every dual algebra of a Kripke frame validates it. Therefore, we consider dual algebras of neighborhood frames defined on the set of Q-filters. Then we show a model existence theorem for predicate modal logics: For any predicate modal logic L, whether it is normal or not, there exists a neighborhood model $\mathcal{M}$ with constant domains which satisfies that $\phi \in \mathrm{L}$ iff $\mathcal{M} \models \phi$ for any formula $\phi$. We also show that the least predicate modal logics which are axiomatized by some of the axioms

$$
\square \top, \square(p \wedge q) \supset \square p \wedge \square q, \square p \wedge \square q \supset \square(p \wedge q)
$$


are complete with respect to the corresponding classes of neighborhood frames with constant domains. The completeness of these logics are already given by ArlóCosta and Pauit in [2], but we give another algebraic proof. As is shown in [2], the Barcan formula is not valid in the class of neighborhood frames with constant domains. Indeed, the least predicate extension of $\mathrm{K}$, in which $\mathrm{BF}$ is not provable, is complete with respect to a class of neighborhood frames with constant domains. See [1] for more about the relation between the Barcan formula and neighborhood frames. We also apply the representation theorem to infinitary modal logics, and show the model existence theorem for all infinitary modal logics. As a corollary, we give completeness theorem for some infinitary modal logics, including the least infinitary extension $\mathrm{K}_{\omega_{1}}$ of $\mathrm{K}$. The completeness theorem of $\mathrm{K}_{\omega_{1}}$ is already given by Minari in [7, but we extend it and give the same algebraic proof as predicate modal logics.

The outline of the paper is as follows: In Section 2, we recall basic definitions. In Section 3, we present an extension of the Jónsson-Tarski representation. In Section 4, we show model existence theorem and completeness theorem for monotonic predicate modal logics. In Section 5 and Section 6, we discuss non-monotonic predicate modal logics and infinitary modal logics, respectively.

\section{Preliminaries}

In this section, we recall basic definitions.

Let $\langle W, \leq\rangle$ be a partially ordered set. For any $X \subseteq W$, we write $\uparrow X$ for the upward closure of $X$. That is,

$$
\uparrow X=\{w \in W \mid \exists x \in X(x \leq w)\} .
$$

Let $f: A \rightarrow B$ be a mapping from a set $A$ to a set $B$. For any set $X \subseteq A$ and $Y \subseteq B, f[X]$ and $f^{-1}[Y]$ denote the sets

$$
f[X]=\{f(x) \mid x \in X\}, \quad f^{-1}[Y]=\{x \in X \mid f(x) \in Y\},
$$

respectively.

Definition 2.1. A neighborhood frame is a pair $\langle C, \mathcal{V}\rangle$, where $C$ is a non-empty set and $\mathcal{V}$ is a mapping from $C$ to $\mathcal{P}(\mathcal{P}(C)$ ). A neighborhood frame $\langle C, \mathcal{V}\rangle$ is said to be monotonic, topped and closed under finite intersections (cufi, for short), if it satisfies the following conditions respectively:

Monotonic: for any $c \in C, \uparrow \mathcal{V}(c)=\mathcal{V}(c)$;

Topped: for any $c \in C, \mathcal{V}(c)$ includes $C$;

Cufi: for any $c \in C$ and any non-empty finite set $S$, if $S \subseteq \mathcal{V}(c)$ then $\bigcap S \in$ $\mathcal{V}(c)$.

Let $Z_{1}=\left\langle C_{1}, \mathcal{V}_{1}\right\rangle$ and $Z_{2}=\left\langle C_{2}, \mathcal{V}_{2}\right\rangle$ be neighborhood frames. A mapping $f: C_{1} \rightarrow C_{2}$ is called a homomorphism of neighborhood frames from $Z_{1}$ to $Z_{2}$, if for any $c \in C_{1}$ and $X \subseteq C_{2}$,

$$
f^{-1}[X] \in \mathcal{V}_{1}(c) \Leftrightarrow X \in \mathcal{V}_{2}(f(c))
$$

holds.

Definition 2.2. An algebra $\langle A ; \vee, \wedge,-, \square, 0,1\rangle$ is called a modal algebra, if its reduct $\langle A ; \vee, \wedge,-, 0,1\rangle$ is a Boolean algebra and $\square$ is a unary operator on $A$. $\mathrm{A}$ modal algebra $A$ is said to be monotonic, topped and cufi, if it satisfies the following conditions, respectively:

Monotonic: for any $x$ and $y$ in $A, \square(x \wedge y) \leq \square x \wedge \square y$;

Topped: $\square 1=1$;

Cufi: for any $x$ and $y$ in $A, \square x \wedge \square y \leq \square(x \wedge y)$. 
Note that each of monotonicity and cufi can be defined by a single equation, and monotonicity is equivalent to

$$
x \leq y \Rightarrow \square x \leq \square y .
$$

Let $A$ and $B$ be modal algebras. A mapping $f: A \rightarrow B$ is called a homomorphism of modal algebras, if $f$ is a homomorphism of Boolean algebras which satisfies

$$
f(\square x)=\square f(x)
$$

for any $x \in A$.

\section{RASIOWA-SIKORSKI LEMMA AND AN EXTENSION OF JÓNSSON-TARSKI REPRESENTATION}

Let $A$ be a Boolean algebra. A non-empty subset $F \subseteq A$ is called a filter of $A$, if it satisfies the following conditions:

(1) $\uparrow F=F$;

(2) $x, y \in A \Rightarrow x \wedge y \in A$, for any $x$ and $y$ in $A$.

A filter is said to be proper, if $0 \notin F$. A proper filter $F$ is said to be prime if $x \vee y \in F$ then either $x \in F$ or $y \in F$, for any $x$ and $y$ in $A$.

Definition 3.1. (Rasiowa-Sikorski [9, 10]). Let $A$ be a Boolean algebra and $S \subseteq$ $\mathcal{P}(A)$. A prime filter $F$ is said to be a $Q$-filter for $S$, if it satisfies that for any $X \in S, \bigwedge X \in F$ whenever $\bigwedge X \in A$ and $X \subseteq F$.

We write $\mathcal{Q F}_{S}(A)$ for the set of all Q-filters for $S$. The following lemma is called the Rasiowa-Sikorski lemma [9, 10].

Lemma 3.2. (Rasiowa-Sikorski [9, 10]). Let $A$ be a Boolean algebra and $S$ a countable subset of $\mathcal{P}(A)$. Then for any $a$ and $b$ in $A$ with $a \not \leq b$, there exists $a$ $Q$-filter $F$ for $S$ such that $a \in F$ and $b \notin F$.

The following two lemmas define a neighborhood frame from a given modal algebra, and vice versa.

Lemma 3.3. Let $A$ be a monotonic modal algebra and $S$ a countable subset of $\mathcal{P}(A)$. Define a pair $J_{S}(A)$ by

$$
J_{S}(A)=\left\langle\mathcal{Q} \mathcal{F}_{S}(A), \mathcal{V}_{A}\right\rangle
$$

where,

$$
\mathcal{V}_{A}(F)=\uparrow\left\{\left\{G \in \mathcal{Q F}_{S}(A) \mid x \in G\right\} \mid \square x \in F\right\}
$$

for any $F$. Then, $J_{S}(A)$ is an monotonic neighborhood frame. Moreover, $J_{S}(A)$ is topped if $A$ is topped, and $J_{S}(A)$ is cufi if $A$ is cufi.

Proof. It is clear from the definition that $J_{S}(A)$ is an monotonic neighborhood frame. Suppose $\square 1=1$ in $A$. Then, for any $F \in \mathcal{Q F}_{S}(A), \square 1 \in F$. Hence,

$$
\mathcal{Q F}_{S}(A)=\{G \mid 1 \in G\} \in \mathcal{V}_{A}(F) \text {. }
$$

Suppose $A$ is cufi. Take any $X$ and $Y$ in $\mathcal{V}_{A}(F)$. Then, there exists $\square x$ and $\square y$ in $F$ such that

$$
\{G \mid x \in G\} \subseteq X,\{G \mid y \in G\} \subseteq Y,
$$

respectively. For any $H \in \mathcal{Q F}_{S}(A)$,

$$
\begin{aligned}
H \in\{G \mid x \in G\} \cap\{G \mid y \in G\} & \Leftrightarrow x \in H, y \in H \\
& \Leftrightarrow x \wedge y \in H \\
& \Leftrightarrow H \in\{G \mid x \wedge y \in G\} .
\end{aligned}
$$

Hence,

$$
\{G \mid x \wedge y \in G\} \subseteq X \cap Y \text {. }
$$


Therefore, $X \cap Y \in \mathcal{V}_{A}(F)$, since $\square x \wedge \square y \leq \square(x \wedge y) \in F$.

Lemma 3.4. Let $Z=\langle C, \mathcal{V}\rangle$ be a neighborhood frame. Define $K(Z)$ by

$$
K(Z)=\left\langle\mathcal{P}(C) ; \cup, \cap, C \backslash-, \square_{Z}, \emptyset, C\right\rangle,
$$

where

$$
\square_{Z} X=\{c \in C \mid X \in \mathcal{V}(c)\}
$$

for any $X \subseteq C$. Then, $K(Z)$ is a modal algebra and if $Z$ is monotonic, topped and cufi, then $K(Z)$ is monotonic, topped and cufi, respectively.

Proof. Suppose $Z$ is monotonic. Then, for any subsets $X$ and $Y$ of $C$, it is clear from the definition that $X \subseteq Y$ implies $\square_{Z} X \subseteq \square_{Z} Y$.

Suppose $Z$ is topped. Then, $C \in \mathcal{V}(c)$ for any $c \in C$. Hence, $\square_{Z} C=C$.

Suppose $Z$ is cufi. Take any subsets $S$ and $T$ of $C$. For any $c \in C$,

$$
\begin{aligned}
c \in \square_{Z} S \cap \square_{Z} T & \Leftrightarrow S \in \mathcal{V}(c), T \in \mathcal{V}(c) \\
& \Rightarrow S \cap T \in \mathcal{V}(c) \\
& \Leftrightarrow c \in \square_{Z}(S \cap T)
\end{aligned}
$$

Hence, $\square_{Z} S \cap \square_{Z} T \subseteq \square_{Z}(S \cap T)$.

We call $K(Z)$ the dual algebra of a neighborhood frame $Z$. Now, we show an extension of the Jónsson-Tarski representation:

Theorem 3.5. Let $A$ be a monotonic modal algebra and $S$ a countable subset of $\mathcal{P}(A)$. Then, a mapping

$$
f: A \rightarrow K \circ J_{S}(A)
$$

defined by

$$
f(x)=\left\{F \in \mathcal{Q F}_{S}(A) \mid x \in F\right\}
$$

is a monomorphism of modal algebras which satisfies that for any $X \in S$, if $\bigwedge X \in$ $A$ then $f(\bigwedge X)=\bigwedge f[X]$

Proof. It is easy to see that $f$ is a homomorphism of Boolean algebras. We show $f$ preserves modal operator. For any $x \in A, f(\square x) \subseteq \square_{J_{S}(A)} f(x)$, since

$$
\begin{aligned}
F \in f(\square x) & \Leftrightarrow \square x \in F \\
& \Rightarrow f(x) \in \mathcal{V}_{A}(F) \\
& \Leftrightarrow F \in \square_{J_{S}(A)} f(x) .
\end{aligned}
$$

We show converse. Suppose $\square x \notin F$. Take any $y \in A$ such that $\square y \in F$. Then, $y \not \leq x$ by monotonicity of $A$. By Rasiowa-Sikorski lemma, there exists $G \in \mathcal{Q F}_{S}(A)$ such that $y \in G$ and $x \notin G$. Hence, $f(y) \nsubseteq f(x)$. Therefore, $f(x) \notin \mathcal{V}_{A}(F)$. Thus, $f$ is a homomorphism of modal algebras.

Suppose $X \in S$ and $\bigwedge X \in A$. For any $F \in \mathcal{Q F}_{S}(A)$,

$$
\begin{aligned}
F \in f(\bigwedge X) & \Leftrightarrow \bigwedge X \in F \\
& \Leftrightarrow X \subseteq F \\
& \Leftrightarrow \forall x \in X(F \in f(x)) \\
& \Leftrightarrow F \in \bigcap_{x \in X} f(x) .
\end{aligned}
$$

Take any $x$ and $y$ in $A$. If $x \not \leq y$, there exists a Q-filter $F$ such that $x \in F$ and $y \notin F$. Hence, $f(x) \neq f(y)$. Therefore, $f$ is a monomorphism of modal algebras. 


\section{Model EXISTENCE FOR PREDICATE MONOTONIC MODAL LOGICS}

The language we consider consists of the following symbols:

(1) a countable set $\mathrm{V}$ of variables;

(2) $\top$ and $\perp$;

(3) logical connectives: $\wedge, \neg$;

(4) quantifier: $\forall$;

(5) for each $n \in \mathbb{N}$, countably many predicate symbols $P, Q, R, \cdots$ of arity $n$;

(6) modal operator $\square$.

The set $\Phi$ of formulas is the smallest set which satisfies:

(1) $\top$ and $\perp$ are in $\Phi$;

(2) if $P$ is a predicate symbol of arity $n$ and $x_{1}, \ldots, x_{n}$ are variables then $P\left(x_{1}, \ldots, x_{n}\right)$ is in $\Phi$

(3) if $\phi$ and $\psi$ are in $\Phi$ then $(\phi \wedge \psi)$ is in $\Phi$;

(4) if $\phi \in \Phi$ then $(\neg \phi)$ and $(\square \phi)$ are in $\Phi$;

(5) if $\phi \in \Phi$ and $x \in \mathrm{V}$ then $(\forall x \phi) \in \Phi$.

The symbols $\vee, \supset, \exists$ are defined in a usual way. We write $\phi \equiv \psi$ and $\diamond \phi$ for abbreviations of $(\phi \supset \psi) \wedge(\psi \supset \phi)$, and $\neg \square \neg \phi$, respectively.

A neighborhood model for predicate modal logic is a four tuple $\langle C, \mathcal{V}, \mathcal{D}, \mathcal{I}\rangle$, where $\langle C, \mathcal{V}\rangle$ is a neighborhood frame, $\mathcal{D}$ is a non-empty set called a domain and $\mathcal{I}$ is a mapping called an interpretation which maps each pair $(c, P)$, where $c \in C$ and $P$ is an $n$-ary predicate symbol, to an $n$-ary relation $\mathcal{I}(c, P) \subseteq \mathcal{D}^{n}$ over $\mathcal{D}$. An assignment $\mathcal{A}$ for $\mathcal{M}$ is a mapping from the set $\mathrm{V}$ of variables to $\mathcal{D}$. For each neighborhood model $\mathcal{M}=\langle C, \mathcal{V}, \mathcal{D}, \mathcal{I}\rangle$ and each assignment $\mathcal{A}$, the valuation $v_{\mathcal{A}}$ of a formula $\phi$ on $\mathcal{M}$ is defined inductively, as follows:

(1) $v_{\mathcal{A}}(\top)=C, v_{\mathcal{A}}(\perp)=\emptyset$;

(2) for any $c \in C$, any predicate $P$ of arity $n$ and any variables $x_{1}, \ldots, x_{n}$, $c \in v_{\mathcal{A}}\left(P\left(x_{1}, \ldots, x_{n}\right)\right)$ iff $\left(\mathcal{A}\left(x_{1}\right), \ldots, \mathcal{A}\left(x_{n}\right)\right) \in \mathcal{I}(c, P)$

(3) $v_{\mathcal{A}}(\phi \wedge \psi)=v_{\mathcal{A}}(\phi) \cap v_{\mathcal{A}}(\psi)$;

(4) $v_{\mathcal{A}}(\neg \phi)=C \backslash v_{\mathcal{A}}(\phi)$;

(5) $v_{\mathcal{A}}(\forall x \phi)=\bigcap_{\mathcal{A}^{\prime} \in A_{x}} v_{\mathcal{A}^{\prime}}(\phi)$, where $A_{x}$ is the set of all assignments for $\mathcal{M}$ which are different from $\mathcal{A}$ only in the value of $x$;

(6) $c \in v_{\mathcal{A}}(\square \phi) \Leftrightarrow v_{\mathcal{A}}(\phi) \in \mathcal{V}(c)$.

Let $\mathcal{M}=\langle C, \mathcal{V}, \mathcal{D}, \mathcal{I}\rangle$ be a neighborhood model and $\mathcal{A}$ an assignment for $\mathcal{M}$. Take any formula $\phi$ and suppose that $\mathcal{A}^{\prime}$ is an assignment for $\mathcal{M}$ such that $\mathcal{A}(x)=$ $\mathcal{A}^{\prime}(x)$ for any $x \in \mathrm{V}$ which occur freely in $\phi$. Then $v_{\mathcal{A}}(\phi)=v_{\mathcal{A}^{\prime}}(\phi)$. Hence, if $\phi$ is a closed formula then $v_{\mathcal{A}}(\phi)=v_{\mathcal{A}^{\prime}}(\phi)$ for any assignments $\mathcal{A}$ and $\mathcal{A}^{\prime}$ for $\mathcal{M}$.

Let $Z=\langle C, \mathcal{V}\rangle$ be a neighborhood frame. For any neighborhood model $\mathcal{M}=$ $\langle C, \mathcal{V}, \mathcal{D}, \mathcal{I}\rangle$, any $c \in C$ and any formula $\phi$, we write $\mathcal{M}, c \models \phi$ if $c \in v_{\mathcal{A}}(\phi)$ for any assignment $\mathcal{A}$ for $\mathcal{M}$. If $\mathcal{M}, c \models \phi$ for any $c \in C$, we write $\mathcal{M} \models \phi$. If $\mathcal{M} \models \phi$ for any domain $\mathcal{D}$ and any interpretation $\mathcal{I}$, we write $Z \models \phi$. Let $C$ be a class of neighborhood frames. For any formula $\phi$, we write $C \models \phi$ if $Z \models \phi$ for any $Z \in C$.

A set $\mathrm{L}$ of formulas is called a predicate modal logic, if it contains classical predicate logic as a subset and closed under substitution, modus ponens and the following two inference rules:

(1) for any $\phi \in \mathrm{L}$ and $x \in \mathrm{V}$, if $\phi \in \mathrm{L}$ then $\forall x \phi \in \mathrm{L}$;

(2) for any $\phi$ and $\psi$ in $\mathrm{L}$, if $\phi \equiv \psi \in \mathrm{L}$ then $\square \phi \equiv \square \psi \in \mathrm{L}$.

A predicate modal logic $\mathrm{L}$ is said to be monotonic, topped and cufi, if $\square(p \wedge q) \supset$ $\square p \wedge \square q \in \mathrm{L}, \square \top \in \mathrm{L}$ and $\square p \wedge \square q \supset \square(p \wedge q) \in \mathrm{L}$, respectively. If a modal logic $\mathrm{L}$ is topped, then the necessitation rule is admissible in $\mathrm{L}$. A predicate modal logic $\mathrm{L}$ 
is said to be normal if it is monotonic, topped and cufi. The least predicate normal modal logic is the least predicate extension of $\mathrm{K}$, which we write $\mathrm{K}_{*}$.

Let $\mathrm{L}$ be a predicate modal logic. Define a binary relation $\cong_{\mathrm{L}}$ on the set $\Phi$ of all formulas by $\phi \cong_{\mathrm{L}} \psi$ iff $\phi \equiv \psi \in \mathrm{L}$. Then, $\Phi / \cong_{\mathrm{L}}$ is a well-defined modal algebra, which is called the Lindenbaum algebra of $\mathrm{L}$. For any formula $\phi$, we write $|\phi|$ for the equivalence class of $\phi$. We claim that for any formula $\phi$ and any $x \in \mathrm{V},|\forall x \phi|=$ $\bigwedge_{y \in \mathrm{V}}|\phi[x:=y]|$ holds in the Lindenbaum algebra of L. Since $\forall x \phi \supset \phi[x:=y]$ is in $\mathrm{L}$ for any $y \in \mathrm{V}$, the set of all equivalence classes of the shape $|\phi[x:=y]|$ has $|\forall x \phi|$ as its lower bound. We show $|\forall x \phi|$ is the greatest lower bound of this set. Suppose that $|\psi|$ is a lower bound of this set. Take a variable $z$ which does not occur in $\psi$ and $\phi$. Then, $\forall z(\psi \supset \phi[x:=z]) \in \mathrm{L}$, since $\psi \supset \phi[x:=z] \in \mathrm{L}$. As $z$ does not occur in $\psi, \psi \supset \forall z(\phi[x:=z]) \in \mathrm{L}$. Hence, $|\psi| \leq|\forall z(\phi[x:=z])|=|\forall x \phi|$. This complete the proof of the claim. The following is the model existence theorem for predicate monotonic modal logic.

Theorem 4.1. For any predicate monotonic modal logic $\mathrm{L}$, there exists a monotonic neighborhood model $\mathcal{M}$ with constant domains which satisfies that

$$
\phi \in \mathrm{L} \Leftrightarrow \mathcal{M} \models \phi
$$

for any closed formula $\phi$. If $\mathrm{L}$ is topped and/or cufi, there exists a topped and/or cufi monotonic neighborhood model $\mathcal{M}$ with constant domains which satisfies (4.1), respectively.

Proof. Let $A_{\mathrm{L}}$ be the Lindenbaum algebra of L. Define $S \subseteq A_{\mathrm{L}}$ by

$$
S=\{|\forall x \phi| \mid x \in \mathrm{V}, \phi \in \Phi\} .
$$

Define a neighborhood model $\mathcal{M}=\langle C, \mathcal{V}, \mathcal{D}, \mathcal{I}\rangle$ by $\langle C, \mathcal{V}\rangle=J_{S}\left(A_{\mathrm{L}}\right)$, that is, the neighborhood frame obtained from $A_{\mathrm{L}}$ in Lemma 3.3. $\mathcal{D}=\mathrm{V}$ and

$$
\left(x_{1}, \ldots, x_{n}\right) \in \mathcal{I}(F, P) \Leftrightarrow\left|P\left(x_{1}, \ldots, x_{n}\right)\right| \in F
$$

for any variables $x_{1}, \ldots, x_{n}$, any $n$-ary predicate $P$ and any Q-filter $F$ for $S$. Define an assignment $\mathcal{A}$ for $\mathcal{M}$ by $\mathcal{A}(x)=x$ for any $x \in \mathrm{V}$. Then, for any formula $\phi$, it follows that $v_{\mathcal{A}}(\phi)=f(|\phi|)$, where $f$ is a monomorphism given in Theorem [3.5] Since $f$ is a monomorphism of modal algebras,

$$
\begin{aligned}
\phi \in \mathrm{L} & \Leftrightarrow|\phi|=1 \text { in } A_{\mathrm{L}} \\
& \Leftrightarrow f(|\phi|)=1 \text { in } K\left(J_{S}\left(A_{\mathrm{L}}\right)\right) \\
& \Leftrightarrow v_{\mathcal{A}}(\phi)=C .
\end{aligned}
$$

If $\phi$ is a closed formula, the last equation is equivalent to $\mathcal{M} \models \phi$. By Lemma 3.3 . $J_{S}\left(A_{\mathrm{L}}\right)$ is topped and/or cufi, whenever $\mathrm{L}$ is topped and/or cufi, respectively.

Corollary 4.2. (Arló-Costa and Pauit [2]). The least (topped and/or cufi) predicate monotonic modal logic is sound and complete with respect to the class of (topped and/or cufi) monotonic neighborhood frames with constant domains, respectively.

Proof. Soundness follows from the leastness and completeness follows from Theorem 4.1.

Hence, $\mathrm{K}_{*}$ is sound and complete with respect to the class $C$ of monotonic topped cufi neighborhood models with constant domains. It is well-known that the Barcan formula $\mathrm{BF}=\forall x \square \phi \supset \square \forall x \phi$ is not a member of $\mathrm{K}_{*}$. Indeed, it is shown in 1] that $C \not \models \mathrm{BF}$. 


\section{Non-MONOTONIC PREDiCATE MODAL LOGICS}

To apply the discussion in Section 4 to predicate non-monotonic modal logic, we change the definition of $J_{S}(A)$ in Lemma 3.3 as follows:

Lemma 5.1. Let $A$ be a non-monotonic modal algebra and $S$ a countable subset of $\mathcal{P}(A)$. Define a pair $\bar{J}_{S}(A)$ by $\bar{J}_{S}(A)=\left\langle\mathcal{Q} \mathcal{F}_{S}(A), \mathcal{V}_{A}\right\rangle$, where,

$$
\mathcal{V}_{A}(F)=\left\{\left\{G \in \mathcal{Q F}_{S}(A) \mid x \in G\right\} \mid \square x \in F\right\}
$$

for any $F$. Then, $\bar{J}_{S}(A)$ is a neighborhood frame, and it is topped if $A$ is topped, and it is cufi if $A$ is cufi

Proof. It is easy to see that is $\bar{J}_{S}(A)$ is a topped neighborhood frame whenever $A$ is topped. Suppose $A$ is cufi. Take any $X$ and $Y$ in $\mathcal{V}_{A}(F)$. Then, there exists $\square x$ and $\square y$ in $F$ such that

$$
X=\{G \mid x \in G\}, Y=\{G \mid y \in G\},
$$

respectively. Since $\square x \wedge \square y \leq \square(x \wedge y) \in F$,

$$
X \cap Y=\{G \mid x \wedge y \in G\} \in \mathcal{V}_{A}(F) .
$$

Then, we obtain the following representation theorem.

Theorem 5.2. Let $A$ be a non-monotonic modal algebra and $S$ a countable subset of $\mathcal{P}(A)$. Then, a mapping

defined by

$$
f: A \rightarrow K \circ \bar{J}_{S}(A)
$$

$$
f(x)=\left\{F \in \mathcal{Q F}_{S}(A) \mid x \in F\right\}
$$

is a monomorphism of modal algebras which satisfies that for any $X \in S$, if $\bigwedge X \in$ $A$ then $f(\bigwedge X)=\bigwedge f[X]$.

Proof. We only show the case for modal operator. For any $x \in A, f(\square x) \subseteq \square f(x)$, since

$$
\begin{aligned}
F \in f(\square x) & \Leftrightarrow \square x \in F \\
& \Rightarrow f(x) \in \mathcal{V}_{A}(F) \\
& \Leftrightarrow F \in \square \bar{J}_{S}(A) \\
& \Leftrightarrow(x) .
\end{aligned}
$$

We show converse. Suppose $\square x \notin F$. Take any $y \in A$ such that $\square y \in F$. Then, $y \not \leq x$ or $x \not \leq x$. By Rasiowa-Sikorski lemma, there exists $G \in \mathcal{Q F}_{S}(A)$ such that $y \in G$ and $x \notin G$ or there exists $H \in \mathcal{Q F}_{S}(A)$ such that $y \notin H$ and $x \in H$, respectively. Hence, $f(y) \neq f(x)$. Therefore, $f(x) \notin \mathcal{V}(F)$.

From Theorem [5.2, the model existence theorem and the completeness theorem for non-monotonic predicate modal logics follow by the same arguments as Section 4. To summarize both monotonic and non-monotonic cases, we have the following:

Theorem 5.3. For any (monotonic and/or topped and/or cufi) predicate modal logic $\mathrm{L}$, there exists a (monotonic and/or topped and/or cufi, respectively) neighborhood model $\mathcal{M}$ with constant domains which satisfies that

$$
\phi \in \mathrm{L} \Leftrightarrow \mathcal{M} \models \phi
$$

for any closed formula $\phi$.

Corollary 5.4. (Arló-Costa and Pauit [2]). The least (monotonic and/or topped and/or cufi) predicate modal logic is sound and complete with respect to the class of (monotonic and/or topped and/or cufi, respectively) monotonic neighborhood frames with constant domains, respectively. 


\section{INFINITARY MODAL LOGICS}

In this section, we apply the arguments in the previous two sections to infinitary modal logics.

The language we consider consists of the following symbols:

(1) a countable set Prop of propositional variables;

(2) $\top$ and $\perp$;

(3) logical connectives: $\bigwedge, \neg$;

(4) modal operator $\square$.

The set $\Phi$ of formulas is the smallest set which satisfies:

(1) $\operatorname{Prop} \subseteq \Phi$;

(2) $\top \in \Phi$ and $\perp \in \Phi$;

(3) if $\Gamma$ is a countable subset of $\Phi$, then $\bigwedge \Gamma \in \Phi$;

(4) if $\phi \in \Phi$ then $(\neg \phi)$ and $(\square \phi)$ are in $\Phi$;

The symbols $\supset$, $\equiv$ and $\diamond \phi$ are defined in the same way as Section 4 For each countable set $\Gamma$ of $\Phi$ we write $\bigvee \Gamma$ for $\neg \bigwedge_{\phi \in \Gamma} \neg \phi$.

Let $\phi$ be any formula. The set $\operatorname{Sub}(\phi)$ of all subformulas of $\phi$ is defined inductively, as follows:

(1) $\operatorname{Sub}(p)=\{p\}$, for all $p \in$ Prop;

(2) $\operatorname{Sub}(T)=\{\top\}$ and $\operatorname{Sub}(\perp)=\{\perp\}$;

(3) $\operatorname{Sub}(\bigwedge \Gamma)=\{\bigwedge \Gamma\} \cup \cup_{\phi \in \Gamma} \operatorname{Sub}(\phi)$;

(4) $\operatorname{Sub}(\square \phi)=\{\square \phi\} \cup \operatorname{Sub}(\phi)$.

A neighborhood model for infinitary modal logic is a triple $\langle C, \mathcal{V}, v\rangle$, where $\langle C, \mathcal{V}\rangle$ is a neighborhood frame and $v$ is a mapping from Prop to $C$, which is called a valuation. For each valuation $v$, the domain Prop is extended to $\Phi$ in the following way:

(1) $v(\top)=C, v(\perp)=\emptyset$;

(2) $v(\bigwedge \Gamma)=\bigcap_{\phi \in \Gamma} v(\phi)$;

(3) $v(\neg \phi)=C \backslash v(\phi)$;

(4) $c \in v(\square \phi) \Leftrightarrow v(\phi) \in \mathcal{V}(c)$.

Let $Z=\langle C, \mathcal{V}\rangle$ be a neighborhood frame. For any neighborhood model $\mathcal{M}=$ $\langle C, \mathcal{V}, v\rangle$ for infinitary modal logic, any $c \in C$ and any formula $\phi$, we write $\mathcal{M}, c \models \phi$ if $c \in v(\phi)$. Other expressions $\mathcal{M} \models \phi, Z \models \phi$ and $C \models \phi$ are defined in the same way as Section 4 .

A set $\mathrm{L}$ of formulas is called an infinitary modal logic, if it contains classical propositional logic as a subset and closed under substitution, modus ponens and the following three inference rules:

(1) if $\bigwedge \Gamma \in \mathrm{L}$ then $\bigwedge \Gamma \supset \phi \in \mathrm{L}$ for any $\phi \in \Gamma$;

(2) if $\psi \supset \phi \in \mathrm{L}$ for any $\phi \in \Gamma$ then $\psi \supset \wedge \Gamma \in \mathrm{L}$;

(3) for any $\phi$ and $\psi$ in $\mathrm{L}$, if $\phi \equiv \psi \in \mathrm{L}$ then $\square \phi \equiv \square \psi \in \mathrm{L}$.

An infinitary modal logic $\mathrm{L}$ is said to be monotonic, topped and cufi, if $\square(p \wedge q) \supset$ $\square p \wedge \square q \in \mathrm{L}, \square \top \in \mathrm{L}$ and $\square p \wedge \square q \supset \square(p \wedge q) \in \mathrm{L}$, respectively. An infinitary modal logic $\mathrm{L}$ is said to be normal if it is monotonic, topped and cufi. We write $\mathrm{K}_{\omega_{1}}$ for the least normal infinitary modal logic, which is the least infinitary extension of $\mathrm{K}$.

Let $L$ be an infinitary modal logic. As we have conjunction of any countable sets of formulas, there exist uncountably many elements of the shape $|\bigwedge \Gamma|$ in the Lindenbaum algebra $A_{\mathrm{L}}$ of $\mathrm{L}$. On the other hand, the monomorphism given in Theorem 3.5 and 5.2 can preserve only countably many infinitary meets. However, we have a restricted version of the model existence theorem, as follows: 
Theorem 6.1. Let $U$ be a countable set of formulas. For any infinitary (monotonic and/or topped and/or cufi) modal logic L, there exists a (monotonic and/or topped and/or cufi, respectively) neighborhood model $\mathcal{M}$ which satisfies that

$$
\phi \in \mathrm{L} \Leftrightarrow \mathcal{M} \models \phi
$$

for any $\phi \in U$.

Proof. We only show the case that $\mathrm{L}$ is monotonic. Let $A_{U}$ be the subalgebra of $A_{\mathrm{L}}$ generated by the set

$$
\bigcup_{\phi \in U}\{|\psi| \mid \psi \in \operatorname{Sub}(\phi)\}
$$

Since $U$ is countable, so is $A_{U}$. Define a subset $S$ of $\mathcal{P}\left(A_{U}\right)$ by

$$
S=\left\{\{|\phi| \mid \phi \in \Gamma\}|| \bigwedge \Gamma \mid \in A_{U}\right\} .
$$

Then, $S$ is also countable. We claim that for any $|\wedge \Gamma| \in A_{U}$,

$$
|\bigwedge \Gamma|=\bigwedge_{\phi \in \Gamma}|\phi|
$$

For any $\phi \in \Gamma,|\bigwedge \Gamma| \leq|\phi|$ holds, since $\bigwedge \Gamma \supset \phi \in \mathrm{L}$ by definition of infinitary modal logic. Hence, $|\bigwedge \Gamma|$ is a lower bound of the set $\{|\phi| \mid \phi \in \Gamma\}$. Suppose that $|\psi| \in A_{U}$ is a lower bound of this set. Then, $\psi \supset \phi \in \mathrm{L}$, for any $\phi \in \Gamma$. By definition of infinitary modal logic, $\psi \supset \wedge \Gamma \in \mathrm{L}$. Therefore, $|\psi| \leq|\wedge \Gamma|$. This complete the proof of the claim. Define a neighborhood model $\mathcal{M}=\langle C, \mathcal{V}, v\rangle$ by $\langle C, \mathcal{V}\rangle=J_{S}\left(A_{U}\right)$ and

$$
F \in v(p) \Leftrightarrow|p| \in F .
$$

By the same argument as in the proof of Theorem 4.1, we obtain that

$$
\phi \in \mathrm{L} \Leftrightarrow \mathcal{M} \models \phi
$$

for any $|\phi| \in A_{U}$, and that $\mathcal{M}$ is monotonic.

As a corollary, we obtain an extension of the completeness theorem for $\mathrm{K}_{\omega_{1}}$ given by Minari [7], as follows:

Corollary 6.2. The least (monotonic and/or topped and/or cufi) infinitary modal logic is sound and complete with respect to the class of (monotonic and/or topped and/or cufi, respectively) monotonic neighborhood frames, respectively.

Proof. We only show that the logic $\mathrm{K}_{\omega_{1}}$ is sound and complete with respect to the class $C$ of monotonic topped cufi neighborhood frames. Suppose $\phi \in \mathrm{K}_{\omega_{1}}$. Then, $C \models \phi$, by the leastness of $\mathrm{K}_{\omega_{1}}$. Suppose $\phi \notin \mathrm{K}_{\omega_{1}}$. Let $U=\{\phi\}$. By Theorem 6.1, there exists a monotonic topped cufi neighborhood model $\mathcal{M}$ such that $\mathcal{M} \forall \phi$. Hence, $C \not \models \phi$.

It is known that the formula

$$
\mathrm{BF}_{\omega_{1}}=\bigwedge_{i \in \omega} \square p_{i} \supset \square \bigwedge_{i \in \omega} p_{i},
$$

that is, an infinitary translation of the Barcan formula BF, is valid in every Kripke model. However, $\mathrm{BF}_{\omega_{1}}$ is not a member of $\mathrm{K}_{\omega_{1}}$. Hence, $\mathrm{K}_{\omega_{1}}$ is Kripke incomplete [13, 7]. In contrast, $\mathrm{BF}_{\omega_{1}}$ is refuted in a neighborhood frame [7]. The counter example in 7 is given by a multi-relational Kripke frame, but it is easy to obtain an equivalent neighborhood frame from it. 


\section{REFERENCES}

[1] Horacio Arló-Costa. First-order extensions of classical systems of modal logic; the role of the Barcan schemas. Studia Logica, 71:87-118, 2002.

[2] Horacio Arló-Costa and Eric Pacuit. First-order classical modal logic. Studia Logica, 84(2):171-210, 2006.

[3] Patrick Blackburn, Maarten de Rijke, and Yde Venema. Modal Logic. Cambridge, third edition, 2001.

44] Alexander Chagrov and Michael Zakharyaschev. Modal Logic. Oxford University Press, 1997.

[5] Kosta Došen. Duality between modal algebras and neighbourhood frames. Studia Logica, 48:219-234, 1989.

[6] Bjarni Jónsson and Alfred Tarski. Boolean algebras with operators I. American Journal of Mathematics, 73:891-931, 1951.

[7] Pierluigi Minari. Some remarks on the proof-theory and the semantics of infinitary logic. In Reinhard Kahle, Thomas Strahm, and Thomas Studer, editors, Advances in Proof Theory, pages 291-318. Springer, 2016.

[8] Hiroakira Ono and Cecylia Rauszer. On an algebraic and Kripke semantics for intermediate logics. In Universal Algebra and Applications, pages 431-438. PWN-Polish Scientific Publishers, 1982.

[9] Helena Rasiowa and Roman Sikorski. A proof of the completeness theorem of Gödel. Fundamenta Mathematicae, 37:193-200, 1950.

[10] Helena Rasiowa and Roman Sikorski. The Mathematics of Metamathematics. PWN-Polish Scientific Publishers, 1963.

[11] Cecylia Rauszer and Bogdan Sabalski. Representation theorem for distributive pseudoBoolean algebra. Bulletin of the Section of Logic, 3:3/4:17-21, 1974.

[12] Cecylia Rauszer and Bogdan Sabalski. Notes on the rasiowa-sikorski lemma. Bulletin of the Section of Logic, 4/3:109-113, 1975.

[13] Yoshihito Tanaka. Kripke completeness of infinitary predicate multi-modal logics. Notre Dame Journal of Formal Logic, 40:326-340, 1999.

[14] Yoshihito Tanaka and Hiroakira Ono. The Rasiowa-Sikorski lemma and Kripke completeness of predicate and infinitary modal logics. In Michael Zakharyaschev, Krister Segerberg, Maarten de Rijke, and Heinrich Wansing, editors, Advances in Modal Logic, volume 2, pages 419-437. CSLI Publication, 2000. 